\section{JTI}

JOURNAL OF

TRAUMA AND INJURY

\title{
Delayed Ascending Aorta Replacement in Blunt Chest Trauma with Aortic Injury
}

\author{
Shin-Ah Son, M.D., Gun-Jik Kim, M.D., Young Woo Do, M.D., Tak-Hyuk Oh, M.D. \\ Department of Thoracic and Cardiovascular Surgery, Kyungpook National University Hospital, \\ Daegu, Korea
}

Received: December 27, 2017

Revised: January 16, 2018

Accepted: January 31, 2018

\section{Correspondence to}

Tak-Hyuk Oh, M.D.

Department of Thoracic and Cardiovascular Surgery, Kyungpook National University Hospital, 130 Dongdeok-ro, Jung-gu,

Daegu 41944, Korea

Tel: $+82-53-200-5665$

Fax: +82-53-426-4765

E-mail: ohhidongii@gmail.com
Ascending aortic injury after blunt chest trauma is an emergency condition that requires urgent diagnosis and treatment. The authors report the case of a patient with traumatic ascending aortic injury who received ascending aorta replacement under cardiopulmonary bypass after failure of primary repair.

Keywords: Aorta; Multiple trauma; Cardiopulmonary bypass

\section{INTRODUCTION}

For many years, traumatic aortic injury (TAI) has been considered a highly lethal lesion and potential cause of death in blunt chest trauma. Blunt aortic injury (BAI) is associated with significant mortality and high-speed transportation. It was historically estimated that $>75 \%$ of patients experienced prehospital mortality, and of those who arrived at the hospital alive, up to $50 \%$ died within the first 24 hours following injury [1]. The authors report the case of a patient with traumatic ascending aortic injury who received ascending aorta replacement under cardiopulmonary bypass (CPB) after failure of primary repair.

\section{CASE REPORT}

A previously healthy 64-year-old man had an accident on a cultivator handle and visited a local medical center (LMC). His initial blood pressure and heart rate were $80 \mathrm{mmHg}$ and 75 beats per minute, respectively. He was mentally alert. Computed (http://creativecommons.org/licenses/by-nc/4.0/) which permits unrestricted noncommercial use, distribution, and reproduction in any medium, provided the original work is properly cited. 

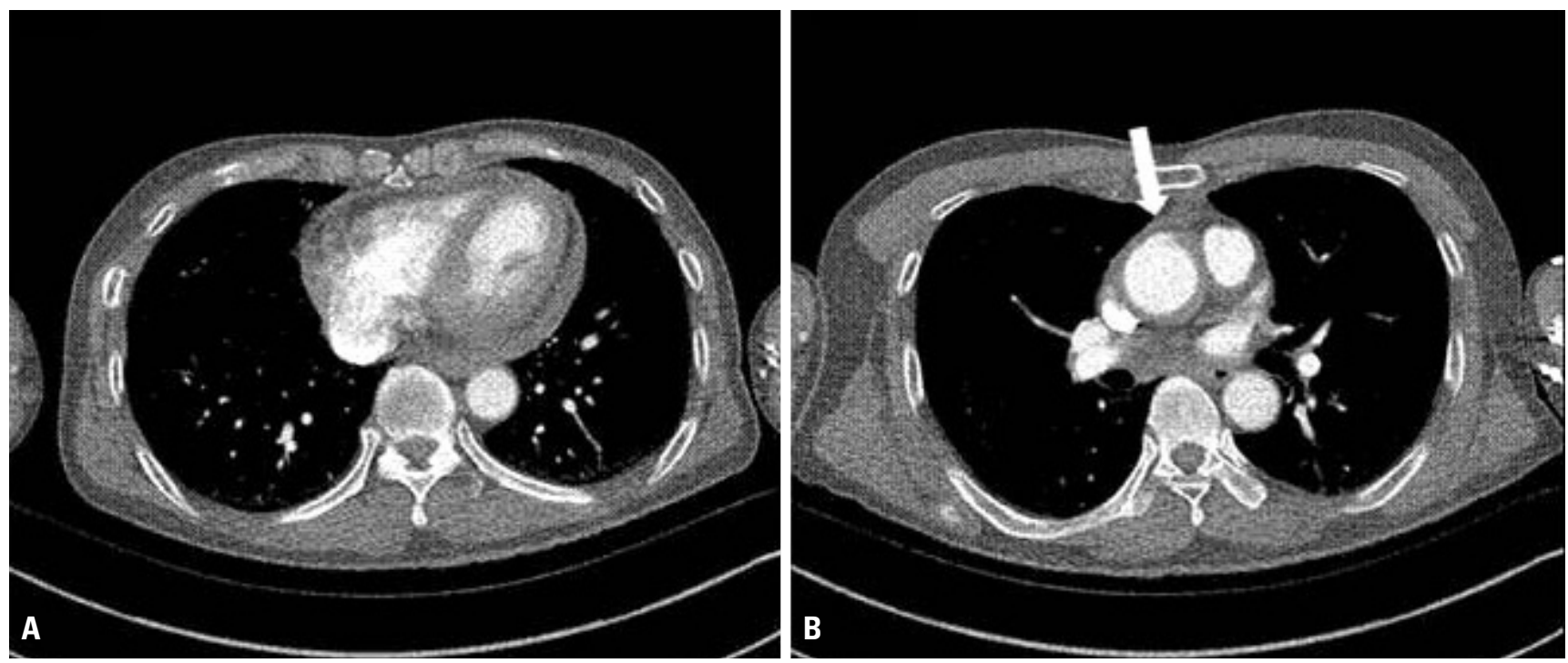

Fig. 1. Initial chest CT showing a small amount of hemopericardium (A) and intimal tearing with hematoma (arrow) on the proximal ascending aorta (B). CT: computed tomography.
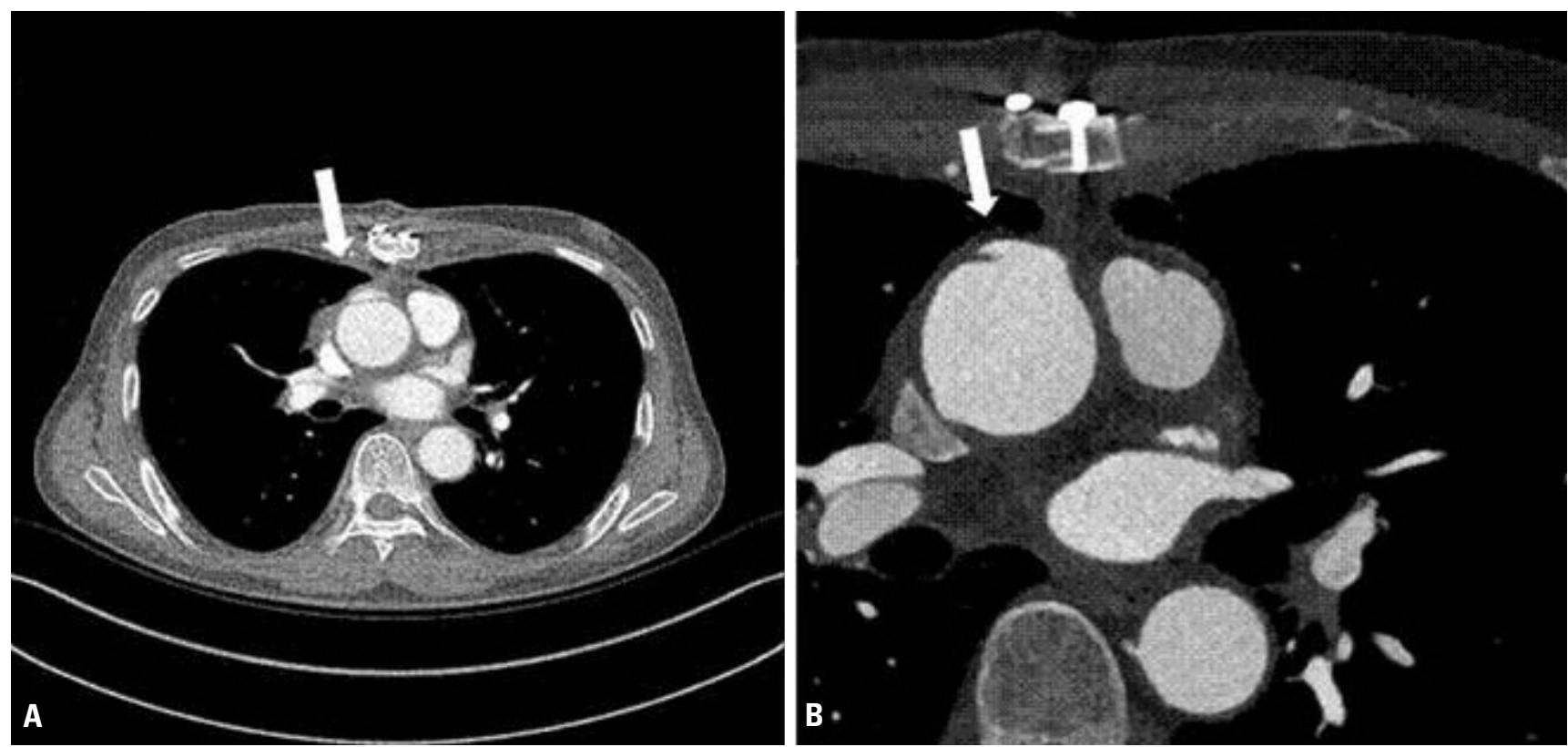

Fig. 2. Follow-up chest CT showing an ascending aortic pseudoaneurysm (arrow) (A), enlarged view (B). CT: computed tomography.

tomography (CT) revealed traumatic hemopericardium, multiple rib fractures, and sternal transverse fracture (Fig. 1). His initial hemoglobin level was $12.5 \mathrm{dg} / \mathrm{dL}$. Electrocardiography revealed normal sinus rhythm.

As the patient had a hemopericardium, emergency exploration was planned. After moving he to the operating room in the LMC, cardiac arrest occurred; hence, an emergency sternotomy was performed. He had a sternal transverse fracture and small amount of hemopericardium. An intimal tearing with pseudoaneurysm was found on the proximal ascending aorta.

With a pledgeted suture, primary closure of the intimal tear was performed, and the sternal transverse fracture was fixated with SternaLock plating. The patient under- 

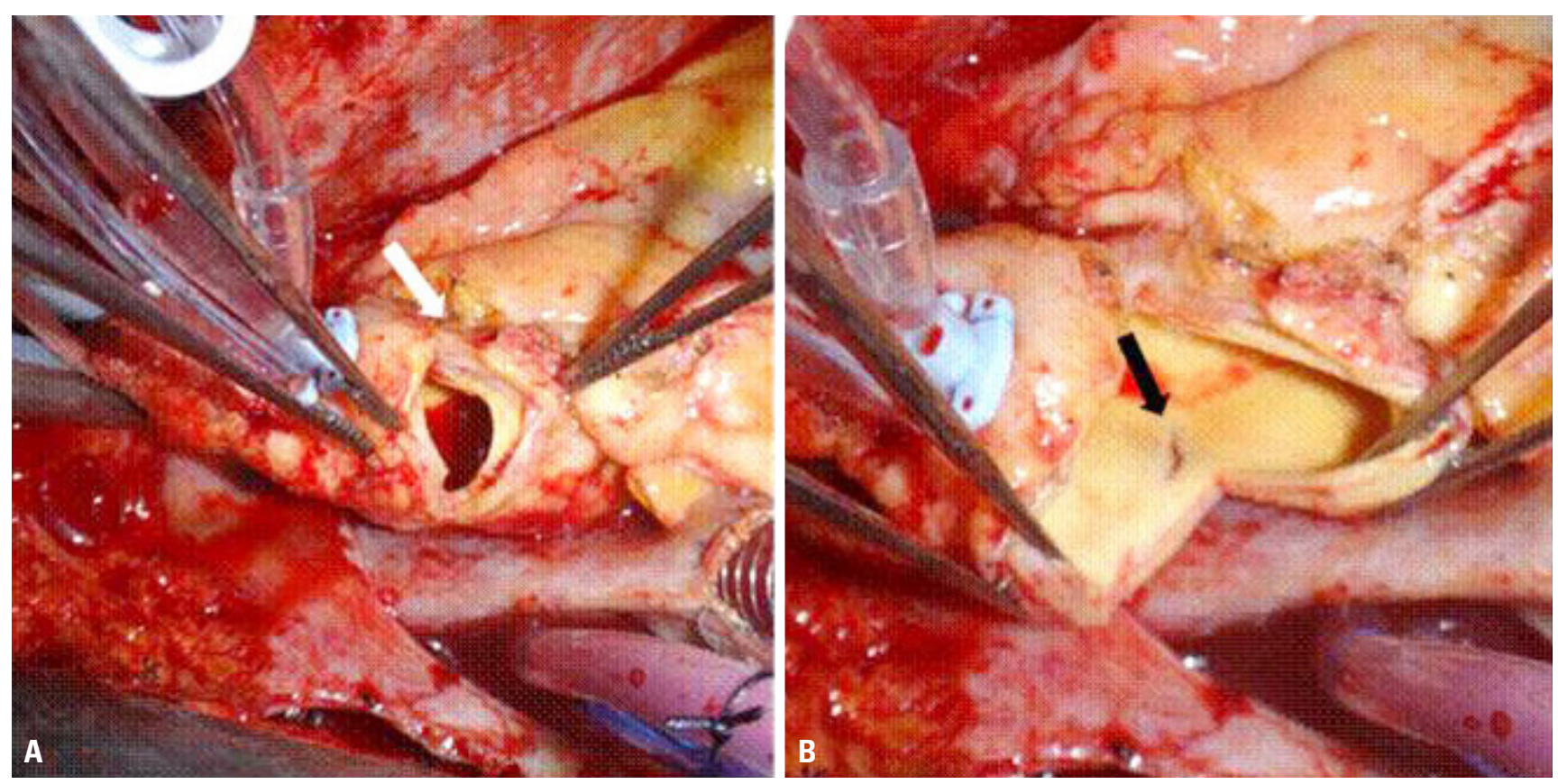

Fig. 3. Intraoperative view of the ascending aortic injury. (A) The arrow indicates the ascending aortic pseudoaneurysm. (B) The arrow shows the transverse tearing of the posterior wall of the ascending aorta, $2 \mathrm{~cm}$ distal to the pseudoaneurysm.

went surgical tracheostomy and was moved to the intensive care unit. Postoperative follow-up CT scan was performed on postoperative day (POD) 2 in the LMC, and the initial ascending aortic injury with pseudoaneurysm formation remained (Fig. 2). The patient's condition was improved, and he was weaned from mechanical ventilation. After POD 20, follow-up CT scan still revealed the ascending aortic pseudoaneurysm, so the patient was transferred to our hospital for further evaluation. The follow-up CT scan obtained in our hospital showed progression of the ascending aortic pseudoaneurysm, and we decided elective ascending aortic replacement under $\mathrm{CPB}$. No significant aortic regurgitation was detected on preoperative transthoracic echocardiography (TTE). The operative finding showed malunion of the sternum due to trauma and the SternaLock plating and severe adhesion in the retrosternal area. The injured aorta was approached through a median sternotomy. CPB was established by arterial cannulation of the ascending aorta near the aortic arch and single right atrial venous cannula. The core temperature was allowed to drift to $32^{\circ} \mathrm{C}$. Pseudoaneurysmal formation was found near the proximal ascending aorta and ascending aorta posterior wall transverse tearing, 2 $\mathrm{cm}$ distal to the pseudoaneurysm (Fig. 3). For the aortic injuries, circumferential removal and ascending aorta replacement with a $26-\mathrm{mm}$ Hemashield graft were performed. Interrupted horizontal mattress pledgeted $4 / 0$ Prolene sutures were used to ensure full-thickness aortic integrity. No intraoperative event occurred on bypass weaning, so the operation was completed. The postoperative vital sign was stable, and the patient was extubated as usual, without adverse event. The patient was moved to the general ward on POD 2 and discharged on POD 11 without complications (Fig. 4).

\section{DISCUSSION}

BAI is associated with significant mortality and highspeed transportation. TAI represents $15 \%$ of motor vehicle-related deaths, with death occurring at the scene in $85 \%$ of the cases [2]. BAI and TAI are highly lethal as most victims die at the scene of the accident or during transportation [3]. Thus, BAI is a life-threatening condition that requires prompt diagnosis and management.

BAI mostly occurs at sites of aortic tethering, aortic 

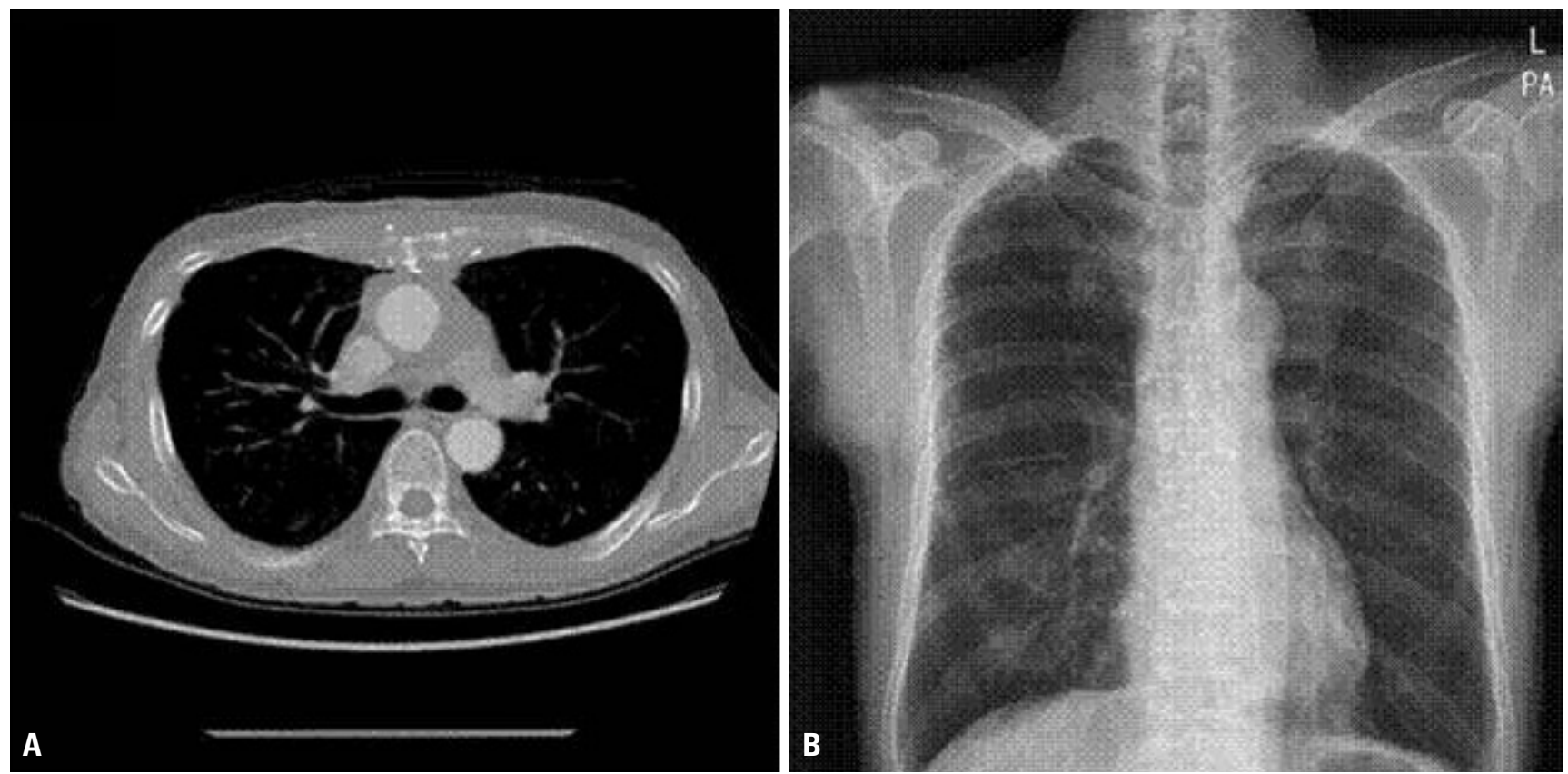

Fig. 4. Postoperative chest CT (A) and radiography images (B). CT: computed tomography.

root, isthmus, and diaphragmatic hiatus. Injuries at the isthmus and abdominal aorta are the most commonly observed in surgery [2]. Traumatic injuries of the ascending aorta and aortic arch are more common in penetrating trauma, whereas injuries of the isthmus and descending aorta are more frequent in blunt trauma [3]. According to the American Association for the Surgery of Trauma (AAST), the ascending thoracic aorta/aortic arch was involved in 7\% of cases in the AAST1 study [1] and in 5.4\% of cases in the AAST2 study [4]. More recently, Teixeira et al. [5] reported a series of 104 subjects with fatal blunt trauma with associated injuries to the ascending aorta and aortic arch, of which $3 \%$ were located at the ascending aorta and $11 \%$ at the arch [5].

BAI presents as a wide range of pathologies, from small intimal defects to full-thickness aortic transections with rupture. According to the classification proposed, Azizzadeh et al. [6] suggested type I (intimal tear), type II (intramural hematoma), type III (pseudoaneurysm), or type IV aortic injuries (rupture). Types I and II were considered low-risk injuries; and types III and IV, high-risk injuries. Diagnosis was based on TTE and/or CT scan.

Clinically, BAI may be missed at the initial post-trauma assessment owing to the lack of suspicion of cardiac involvement. In addition, other coexistent injuries such as fractured ribs and pulmonary contusion with the same chief complaints may mask the manifestations of cardiac injury. Mosquera et al. [7] reported that significant aortic regurgitation (AR) developed in $12 \%$ of patients with injuries at the ascending aorta as a consequence of the thoracic trauma. Proposed mechanisms that contribute to BAI include shearing forces, rapid deceleration, hydrostatic forces, and osseous pinch [8]. Rapid deceleration in the anteroposterior and lateral directions has been shown to be sufficient to result in cardiac displacement, producing torsion and shearing forces against the aorta at levels of relative immobility, mainly the ligamentum arteriosum, aortic root, and diaphragm [9]. A combination of compression and upward thrust of the heart, which also involves shear and torsion, has been suggested [2].

Treatment options for BAI can be divided into three categories as follows: open surgical repair, endovascular repair, and medical management. Traditionally, BAI has been treated with open surgical repair, and this is still the procedure of choice to repair injuries involving the aortic root, ascending aorta, and aortic arch [10]. Endovascular repair has become the initial procedure of choice in some centers for management of BAI involving the aortic isth- 
mus, descending thoracic, or abdominal aorta.

The present case demonstrates a rare presentation of blunt ascending aortic injury. The proximal ascending aorta is an unusual site of transection following blunt trauma. This case is considered BAI due to shearing forces and rapid deceleration on a cultivator handle crush. Harmouche et al. [11] reported ascending aortic injury repair with direct full-layer suture using CPB safely. However, in the present case, simple direct suture without CPB was used, which could increase the risk of pseudoaneurysm because of intolerance to the shear stress on the hemodynamic aorta wall. In addition, the important aorta posterior wall transverse tearing was missed. Especially old age has elevated aortic stiffness, so the aorta could not withstand strong shear stress on the ascending aortic wall, which increases the incidence of devastating aortopathy.

Thus, when patients visit the emergency department with strong impression of ascending aortic injury, they must be transferred to a hospital capable of performing $\mathrm{CPB}$. $\mathrm{CPB}$ allows surgeons to examine the definite injury site of the aorta and perform definite treatment with ascending aorta replacement.

In conclusion, TAI is rare, but blunt ascending aortic injury as the result of crush trauma is extremely uncommon. Ascending aortic injury after blunt chest trauma is an emergency condition that requires urgent diagnosis and treatment, which could not be solved by medical treatment or endovascular surgery. The ascending aorta is a heavily burdened organ due to high pressure. Thus, in ascending aortic injury, ascending aorta replacement under CPB should be performed without hesitation.

\section{REFERENCES}

1. Fabian TC, Richardson JD, Croce MA, Smith JS Jr, Rodman G Jr, Kearney PA, et al. Prospective study of blunt aortic in- jury: Multicenter Trial of the American Association for the Surgery of Trauma. J Trauma 1997;42:374-80; discussion 380-3.

2. Williams JS, Graff JA, Uku JM, Steinig JP. Aortic injury in vehicular trauma. Ann Thorac Surg 1994;57:726-30.

3. Dosios TJ, Salemis N, Angouras D, Nonas E. Blunt and penetrating trauma of the thoracic aorta and aortic arch branches: an autopsy study. J Trauma 2000;49:696-703.

4. Demetriades D, Velmahos GC, Scalea TM, Jurkovich GJ, Karmy-Jones R, Teixeira PG, et al. Blunt traumatic thoracic aortic injuries: early or delayed repair--results of an American Association for the Surgery of Trauma prospective study. J Trauma 2009;66:967-73.

5. Teixeira PG, Inaba K, Barmparas G, Georgiou C, Toms C, Noguchi TT, et al. Blunt thoracic aortic injuries: an autopsy study. J Trauma 2011;70:197-202.

6. Azizzadeh A, Keyhani K, Miller CC 3rd, Coogan SM, Safi HJ, Estrera AL. Blunt traumatic aortic injury: initial experience with endovascular repair. J Vasc Surg 2009;49:1403-8.

7. Mosquera VX, Marini M, Muñiz J, Gulias D, Asorey-Veiga V, Adrio-Nazar B, et al. Blunt traumatic aortic injuries of the ascending aorta and aortic arch: a clinical multicentre study. Injury 2013;44:1191-7.

8. Creasy JD, Chiles C, Routh WD, Dyer RB. Overview of traumatic injury of the thoracic aorta. Radiographics 1997;17:27-45.

9. Burkhart HM, Gomez GA, Jacobson LE, Pless JE, Broadie TA. Fatal blunt aortic injuries: a review of 242 autopsy cases. J Trauma 2001;50:113-5.

10. Cullen EL, Lantz EJ, Johnson CM, Young PM. Traumatic aortic injury: CT findings, mimics, and therapeutic options. Cardiovasc Diagn Ther 2014;4:238-44.

11. Harmouche M, Slimani EK, Heraudeau A, Verhoye JP. Blunt traumatic aortic rupture of the proximal ascending aorta repaired by resection and direct anastomosis. Interact Cardiovasc Thorac Surg 2013;17:739-40. 